\section{Bimatoprost $0.01 \%$ vs bimatoprost 0.03\%: a 12-month prospective trial of clinical and in vivo confocal microscopy in glaucoma patients}

Eye (2014) 28, 506; doi:10.1038/ eye.2014.47

Correction to: Eye (2014) 28, 422-429;

doi:10.1038/eye.2013.304; published online 17 January 2014
M Figus, M Nardi, P Piaggi, M Sartini, G Guidi, L Martini and S Lazzeri
Since the publication of the above article, it has been noted that the colour of the lines in Figure 1 were the wrong way round. The correct figure appears below.

The authors apologise for this error.

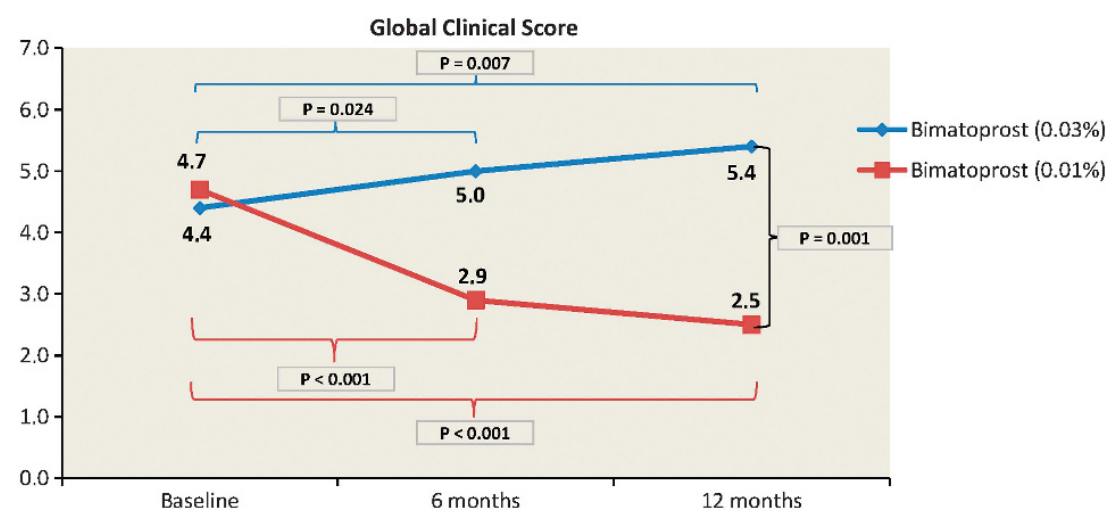

Figure 1 Global clinical score. 